\title{
Crizotinib Versus Conventional Chemotherapy in First-Line Treatment for ALK-Positive Non-Small Cell Lung Cancer: A Systematic Review and Meta- Analysis
}

\author{
Barbara D. Cruz (D) - Mariana M. Barbosa (D) - Lucas L. Torres (D) • \\ Pamela S. Azevedo (D) · Vânia E. A. Silva (D) · Brian Godman · \\ Juliana Alvares-Teodoro (D)
}

Received: February 25, 2021 / Accepted: May 19, 2021 / Published online: June 11, 2021

(c) The Author(s) 2021

\section{ABSTRACT}

Introduction: Lung cancer is the most frequently diagnosed type of cancer and the main cause of death from malignant neoplasms worldwide. One of the most recent discoveries in the context of non-small cell lung cancer (NSCLC) was the mutation of the anaplastic lymphoma kinase receptor (ALK). This genetic alteration is found in approximately $2-5 \%$ of NSCLC patients, and crizotinib was the first

B. D. Cruz $(\bowtie) \cdot$ L. L. Torres · P. S. Azevedo · J. Alvares-Teodoro

Programa de Pós-Graduação Em Medicamentos E Assistência Farmacêutica, Faculdade de Farmácia, Universidade Federal de Minas Gerais, Belo

Horizonte, Brazil

e-mail: bdelanocruz@yahoo.com.br

M. M. Barbosa

Câmara de Regulação Do Mercado de Medicamentos (CMED), Agência Nacional de Vigilância Sanitária (ANVISA), Governo Federal, Brasília, Brazil

V. E. A. Silva

Pontifícia Universidade Católica de Minas Gerais, PUC Minas, Belo Horizonte, Brazil

\section{B. Godman}

Strathclyde Institute of Pharmacy and Biomedical Sciences, University of Strathclyde, Glasgow G4 ORE, UK

B. Godman

School of Pharmacy, Sefako Makgatho Health

Sciences University, Garankuwa, South Africa targeted therapy discovered for its first-line treatment.

Objective: To conduct a systematic review and meta-analysis to estimate the magnitude of the overall survival (OS) and progression-free survival (PFS) from using crizotinib as treatment compared to traditional chemotherapy to guide future decision making.

Methods: PRISMA and Cochrane recommendations were followed using the findings based on studies published in the main international electronic databases. Selection criteria included the following: randomized clinical trials (RCT) or cohort studies that had assessed the efficacy and effectiveness of crizotinib as monotherapy in patients with NSCLC with ALK fusions.

Results: From 2504 publications identified in the literature, only eight publications referring to seven studies met the selection criteria, with high heterogeneity identified between the studies. Overall, there was a significant gain in PFS (HR 0.38; 95\% CI 0.30-0.49; $p<0.00001$ ); however, there was no significant gain in OS (HR 0.68; 95\% CI 0.43-1.08; $p=0.10$ ).

Conclusion: The study highlighted and confirmed that treatment with crizotinib led to clinical improvement in PFS among patients with advanced NSCLC with ALK fusion, as previously reported. However, there was no increase in overall survival in patients with NSCLC with genetic alterations of ALK. This must be considered when reviewing and funding treatments for NSCLC patients with this 
mutation.Keywords: Anaplastic lymphoma kinase; Crizotinib; Effectiveness; Efficacy; Lung cancer; Systematic review

\section{Key Summary Points}

Why carry out this study?

Lung cancer is the main cause of death from malignant cancers worldwide; consequently, there is an appreciable need to improve the care of these patients.

A mutation of the anaplastic lymphoma kinase (ALK) receptor is present in a small minority of patients with non-small cell lung cancer (NSCLC), which offers potential for future drug therapies.

What did the study ask?/What was the hypothesis of the study?

Crizotinib was developed targeting the ALK receptor, and there is a need to assess its effectiveness in managing pertinent NSCLC patients through a systematic review.

\section{What was learned from the study?}

Overall in this systematic review, whilst there was a gain in progression-free survival versus standard of care with crizotinib, this did not translate into an overall survival benefit, which must be considered when reviewing treatment and funding options in these patients.

\section{DIGITAL FEATURES}

This article is published with digital features, including a summary slide, to facilitate understanding of the article. To view digital features for this article, go to https://doi.org/10.6084/ m9.figshare.14605395.

\section{INTRODUCTION}

The World Health Organization (WHO) estimates that one in five men and one in six women worldwide will develop cancer during their lifetime, and that one in eight men and one in 11 women will die from this disease. In this way, cancer has become the main global public health problem, and is among the top four causes of premature death in most countries [1].

The situation is more critical among lowand middle-income countries (LMICs), representing the majority of cancer deaths $[2,3]$, with LMICs accounting for approximately $65 \%$ or more of all cancer deaths and rising [3-5]. Nevertheless, cancer is also the leading cause of death among high-income countries [6], with cancer accounting for at least $20 \%$ of deaths globally from noncommunicable diseases (NCDs) [4]. New cancer cases are expected to reach 29.5 million by 2040, up from 18.1 million currently $[7,8]$. Alongside this, the global outlay on oncology medicines is expected to increase to $\$ 237$ billion by 2024 [9], assisted by growing prevalence rates, new and often costly cancer medicines with limited health gain in a number of cases, and the appreciable number of companies pursuing the development of new oncology medicines [8-11]. Spending on specialty medicines, including those for cancer, is driving up the cost of medicines, with these therapies envisaged to account for at least 50\% of total medicine costs by 2023, which will place considerable pressure on countries seeking to continue providing universal health care [12]. Among the estimated 18.1 million new cancer cases worldwide in 2018 and 9.6 million cancer deaths, lung cancer was the most frequently diagnosed cancer type $(11.6 \%$ of the total cases) and the main cause of death (18.4\% of total cancer deaths) when considering both genders $[3,6]$. Consequently, there is a constant need to continually re-evaluate the role and value of treatments used in the management of patients with lung cancer.

However, lung cancer is no longer considered a single disease. The histological and genetic variations are now considered critical to 
defining future treatment. Lung cancer is currently divided into two main types: non-small cell lung cancer (NSCLC), representing about $80 \%$ of the new cases, and small cell lung cancer (SCLC) for most of the remainder. WHO further subdivides NSCLC into three main subtypes: adenocarcinoma, squamous cell carcinoma, and large cell carcinoma [13, 14]. Within this scenario, new target therapies have emerged for treating advanced NSCLC, leading to increased expectations among patients and oncologists [15].

Within this context, NSCLC is the solid tumor with the widest variety of potential therapeutic targets, representing both an effective opportunity and a challenge in identifying predictive biomarkers for clinical use. Therefore, we discuss some of the biomarkers that are essential for testing in all patients with this type of solid tumor, to be performed in specimens collected by biopsy in patients with advanced NSCLC. Table 1 shows the main predictive changes to be identified and their test methods.

One of the more recent discoveries of genetic alterations in the field of NSCLC was the ALK mutation, in 2007. Since then, many variants of the rearrangements of the $A L K$ gene have been identified and researched, mapping those that have clinical and pathological importance and are associated with malignant tumor growths in patients with NSCLC. The first oncology medicine to treat NSCLC based on the echinoderm microtubule-associated protein-like 4-anaplastic lymphoma kinase (EML4-ALK) rearrangement was crizotinib, a tyrosine kinase inhibitor capable of blocking the site of action and inhibiting the transmission of tumor replication intracellular signals [16]. Crizotinib was first approved in 2011 by the Food and Drug Administration (FDA) in the USA.

Since then, crizotinib has already been studied in several phase I, II and III trials for first- and second-line treatment, in addition to systematic reviews with or without meta-analysis. This is important, since whilst the occurrence of the ALK mutation is low, currently found in only $2-5 \%$ of diagnosed NSCLC, the high number of lung cancer patients worldwide makes EML4-ALK an important genetic biomarker [16].

In order to enhance the practice of evidencebased medicine, especially with the envisaged continued growth in expenditure on medicines for patients with cancer, a systematic review with meta-analysis was conducted to synthesize the scientific evidence regarding the use of crizotinib for the first-line treatment of advanced or metastatic NSCLC for patients with a fusion type rearrangement. We are aware that there have been systematic reviews comparing different ALK inhibitors for NSCLC as well as specific subtypes and safety issues [17-21]. However, we wanted to specifically concentrate on crizotinib due to the typically higher price of some newer agents [22, 23]. In addition, there were concerns initially with the cost-effectiveness of crizotinib alleviated by discounts and rebates [24, 25]. Consequently, we wanted to provide baseline data for any subsequent evaluation of therapies for NSCLC patients with

Table 1 NSCLC biomarkers testing methodology

\begin{tabular}{lll}
\hline Biomarker & Alteration & Methodology \\
\hline$E G F R$ & Mutation & PCR: sanger, real-time PCR and NGS \\
$A L K$ & Rearrangement & IHC, FISH and NGS \\
ROS1 & Rearrangement & IHC (screening), FISH and NGS \\
BRAF V600 & Mutation & PCR: sanger, real-time PCR and NGS \\
PD-L1 & Overexpression & IHC \\
\hline
\end{tabular}

EGFR epidermal growth factor receptor, $A L K$ anaplastic lymphoma kinase receptor, FISH fluorescence in situ hybridisation, $I H C$ immunohistochemistry, NGS next-generation sequencing, $P C R$ polymerase chain reaction, $P D-L 1$ programmed death ligand-1 
ALK mutations, especially with Brazil starting to consider new reimbursement approaches such as risk-sharing arrangements to help fund new premium-priced therapies [26]. We believe this is the first systematic review and meta-analysis to summarize the scientific evidence on the use of this drug exclusively in the first line of treatment for NSCLC ALK+, building on previous publications [27-33].

\section{METHODS}

\section{Search Strategy and Study Identification}

The criteria of the Cochrane Handbook for Systematic Reviews of Interventions (Cochrane) methodological guide were followed. The research registration was submitted and accepted on the International Prospective Register of Systematic Reviews (PROSPERO) platform under the protocol CRD42020164482 [34-37].

Articles were searched from inception to November 2020, and the search was carried out in MEDLINE databases via PubMed (Medical Literature Analysis and Retrieval System Online), EMBASE (Excerpta Medica dataBASE), Cochrane Library and LILACS (Latin American and Caribbean Health Science Literature). A manual search was also undertaken on all references of the included studies in addition to searches on the databases of ClinicalTrials.gov, EU clinical trials, the records of clinical studies in Brazil-Brazilian Registry of Clinical Trials (ReBEC), and the WHO International Clinical Trials Registry Platform.

The following words were used in the search: crizotinib, cisplatin, carboplatin, etoposide, mitomycin $\mathrm{C}$, vinblastine, vinorelbine, gemcitabine, docetaxel, paclitaxel, pemetrexed, bevacizumab, alone or in combination as a structured search, and for those, descriptors were used that included a combination of keywords, synonyms, and controlled descriptors: health sciences descriptors (DECs) and Medical Subject Heading $(\mathrm{MeSH})$, free terms, truncated terms and planned text according to each type of database. In the PubMed and EMBASE databases, the controlled descriptors $\mathrm{MeSH}$ and Emtree were used, respectively, in order to locate and retrieve all relevant available evidence.

Our study protocol strictly followed the recommendations of the Preferred Reporting Items for Systematic Reviews and Meta-Analyses (PRISMA) statement [38, 39]. This article is based on previously conducted studies and does not contain any new studies with human participants or animals performed by any of the authors.

\section{Selection Criteria and Abstract Screening}

The selected studies were entered into Rayyan $\mathrm{QCRI}^{\circledR}$, allowing two independent and blinded reviewers to screen the titles and abstracts of all included articles. Subsequently, the full text of the selected papers was read by both reviewers (LLT, PSA). Cases of conflict were resolved by a third independent reviewer (BDC).

Studies were eligible if they fulfilled the criteria as randomized controlled trials (RCT) or cohort studies and had assessed the efficacy and/or effectiveness of crizotinib as monotherapy in patients with NSCLC with $A L K$ fusions. Studies were excluded from the systematic review if they did not include the following characteristics: (a) lung cancer, (b) first-line treatment for stage III and IV, (c) a comparator arm with chemotherapy, and (d) an RCT or observational cohort study. We included published studies in a number of languages including English, Portuguese, Spanish and French for completeness.

Trials in more than one database were counted as duplicates. However, publications other than the same trial were counted as one unit and two reports.

\section{Full-Text Screening and Data Extraction}

The extracted characteristics and qualitative information from the studies included the following: first author, year of publication, study publication date, publication language, type of study design, location, and whether to include in the systematic review and meta-analysis, with description of the exclusion criteria and characteristics of the patients (gender, age, 
performance status, smoking history, histology, disease stage), along with the general characteristics of the trials (design, inclusion and exclusion criteria, treatments of the intervention and control groups, evaluated outcomes) and their results. The results of the trials were reported according to the outcome and in terms of overall survival (OS) or progression-free survival (PFS) as the two main outcome measures typically reported. We are aware that there is controversy surrounding the value of PFS in solid tumors, although this is not always the case [40-44]. Consequently, we included PFS, as it is typically the principal outcome measure reported in most studies with new cancer medicines. The data extraction was performed by the same two independent and blinded reviewers, with the third acting as a judge harmonizing the data.

\section{Data Analysis}

The eligibility criteria adopted in this systematic review were quite sensitive; however, they still resulted in high statistical heterogeneity due to the range of observational studies included. The random model method was subsequently selected as a strategy to mitigate this in carrying out statistical analyses of the different subgroups [34].

Statistical analyses were performed using Review Manager (RevMan) software version 5.3. For continuous variables, the results are expressed in hazard ratios (HR) with their respective $95 \%$ confidence intervals (CI) extracted directly from each original study. To evaluate the heterogeneity between the included studies, the $I^{2}$ statistic was performed, as this was the form of evaluation and guaranteed that the percentage of the total variation between the studies could be attributed to chance. We classified the heterogeneity across studies as low if $25 \%<I^{2} \leq 50 \%$, moderate if $50 \%<I^{2} \leq 75 \%$, and high if $I^{2}>75 \%$. Publication bias was not subsequently analyzed by funnel plots, since this statistical method is recommended when analyzing 10 or more studies, which was not achieved in this situation [34].

\section{RESULTS}

The predefined search strategy identified 2654 potential publications. After removing duplicate studies $(n=527)$, the eligibility criteria were applied to the remaining titles and abstracts. This resulted in 2127 potential studies. Further applying the eligibility criteria resulted in 38 articles considered relevant for reading in full. In the second stage of the process, 30 papers were subsequently excluded for not meeting the following eligibility criteria: language $(n=2)$, one in Czech and one in Chinese; outcomes different from OS and PFS $(n=3)$; absence of a comparator arm as established by the selection criteria $(n=11)$; and study design different from that stipulated for this systematic review and meta-analysis, such as case-control reports and phase II clinical trials $(n=14)$ (Fig. 1$)$.

The search yielded eight publications, and the characteristics of the included studies are shown in Table 2. All included studies recruited patients with advanced-stage NSCLC. The initial dose for crizotinib was $250 \mathrm{mg}$ twice daily in all studies. Treatment efficacy and tumor response were assessed using the Response Evaluation Criteria in Solid Tumors (RECIST) classification [45]. Treatment arms and outcomes of the clinical trials and cohorts included in the systematic review are shown in Tables 3 and 4 , respectively.

\section{Progression-free survival}

With respect to PFS, it was possible to statistically analyze five of the seven studies selected to analyze the data expressed in HRs. The result of the meta-analysis was obtained from 391 patients treated with crizotinib (experimental group) and 419 with conventional chemotherapy (control group).

The meta-analysis of these studies indicated that there was a statistically significant difference between the experimental and control groups for the outcome of PFS, favoring the group of patients who were treated with crizotinib, with a reduction in the risk of progression or death by any cause of $62 \%$ (HR $0.38 ; 95 \%$ CI 


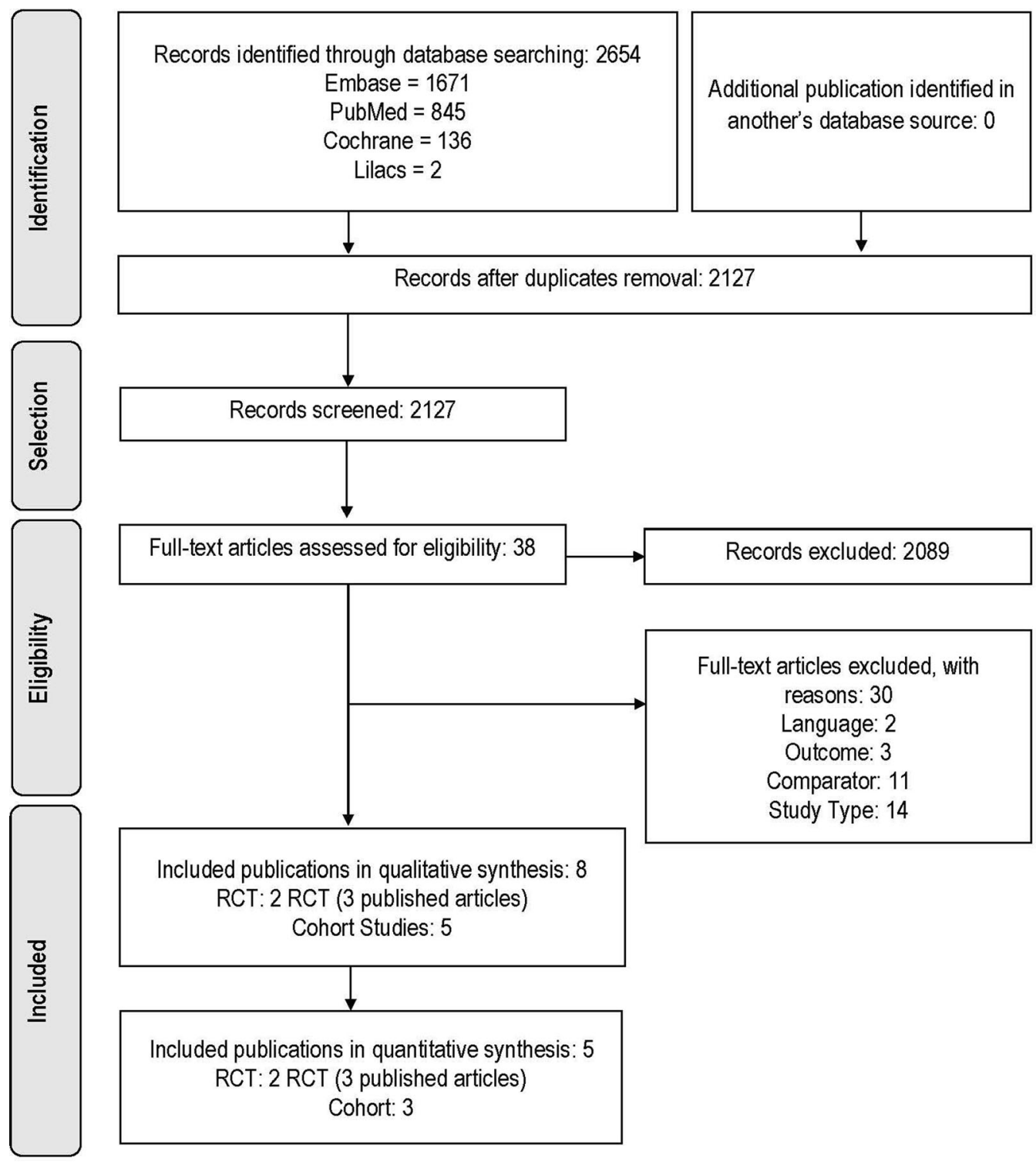

Fig. 1 PRISMA flowchart with the results of the search for systematic reviews and selection of eligible studies

0.30-0.49) in relation to the control arm, and this reduction was statistically significant $(p<0.001)$ (Fig. 2).

As a limitation of this meta-analysis, the result showed moderate heterogeneity $\left(I^{2}=41 \%\right)$; however, it was statistically significant and may be due to both clinical and methodological factors, since the included studies had different designs (RCT and cohorts). However, it is not believed that this heterogeneity is due to the sample population, since criteria such as the age range and gender distribution were well distributed among the groups, as assessed and described in Table 1 where the studies presented a range of age and proportion of similar genders.

When evaluating the subgroup by study type, it was identified that the heterogeneity measured for the RCTs was $I^{2}=0 \%(p=0.60)$, being considered non-significant. This an expected effect since the characteristics of the studies are very similar since, they were conducted by the same sponsor, with similar inclusion criteria, blinded randomization, 


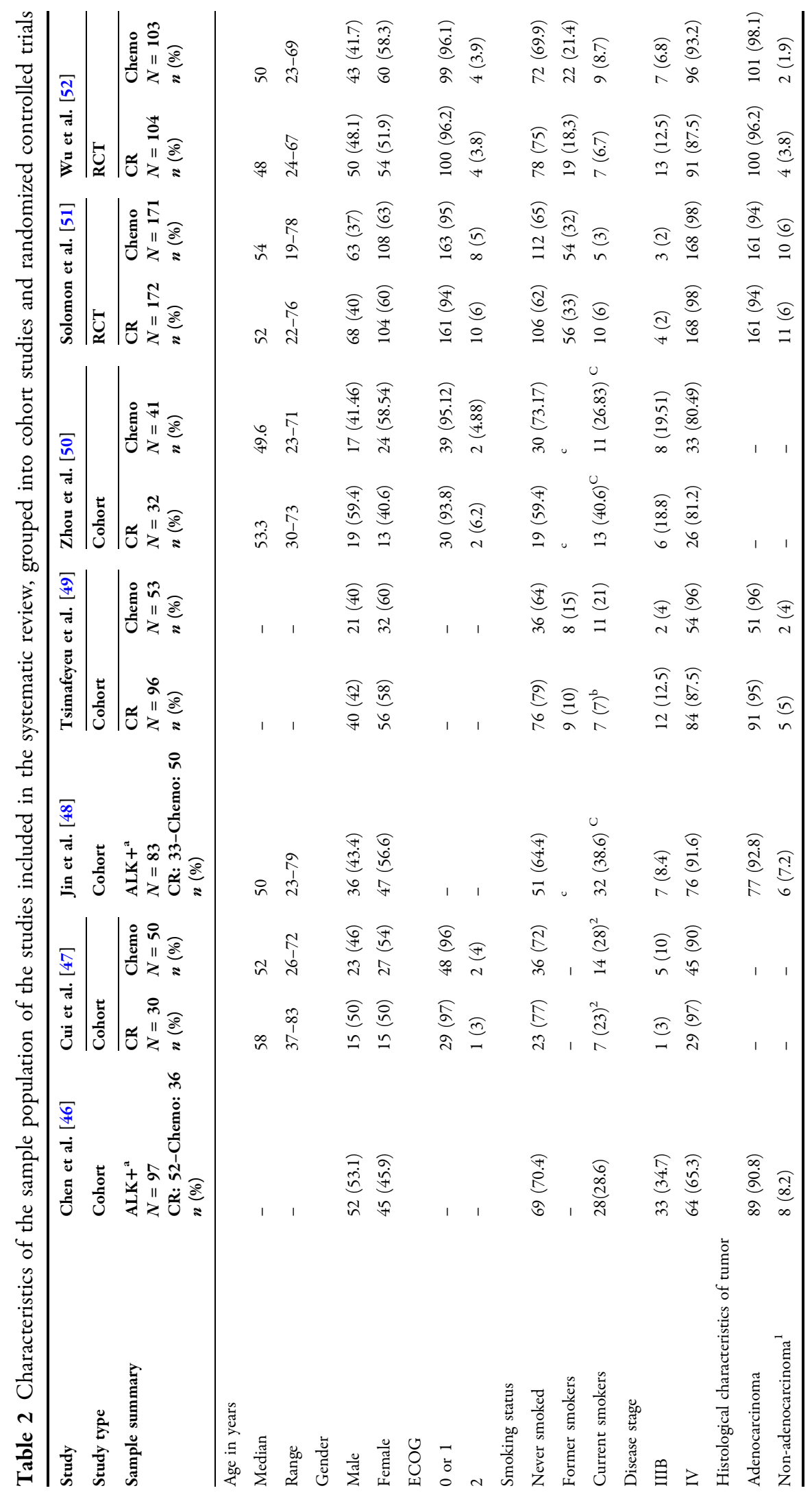




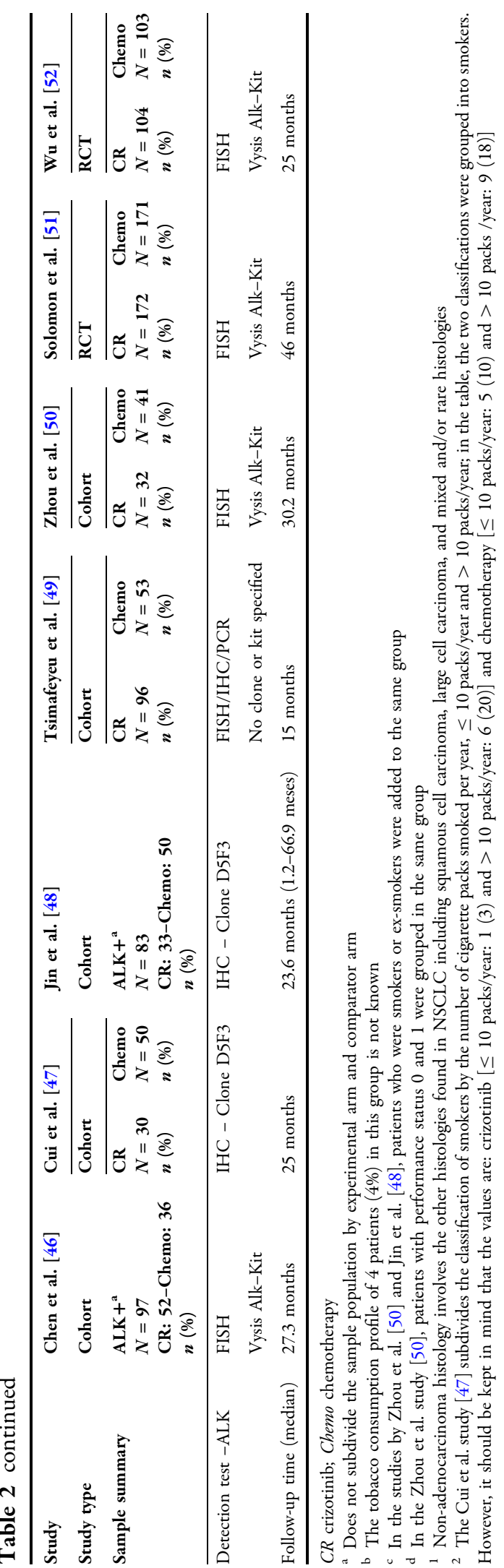

central evaluation for analysis of outcomes, and similar follow-up times.

For the cohort studies, the main differences between the trial designs were considered in terms of intervals for assessing progression, number of patients allocated in each arm, and chemotherapy treatment schedules as comparator arms. This justified the moderate heterogeneity of this subgroup, $I^{2}=52 \%$, however, not significant $(p=0.13)$.

A sensitivity analysis was conducted in the forest plot PFS results to identify the possible cause of the heterogeneity found in the cohort studies. Following the analysis, it was found that the Cui et al. [47] study was the one responsible for causing the increase in heterogeneity. This was probably caused by a longer time interval between evaluation for patients in the crizotinib and control arms. The treatment scheme in the experimental arm (crizotinib) diverged from the one described in the pivotal study which would be continuous use of oral therapy with crizotinib. Another cause for the high heterogeneous result seen could be the higher dose of gemcitabine chosen as treatment (1250 mg/m $\mathrm{m}^{2}$-D1 and D8), docetaxel administered in a different scheme than usual $(75 \mathrm{mg} /$ $\mathrm{m}^{2}-\mathrm{D} 1$ and D8), and only adenocarcinomas NSCLC histology selected as study patients and presented a high grade of non-smokers as a $75 \%$ of the study total population [47].

\section{Overall survival}

Three studies were included for the systematic review relating to OS of patients with NSCLC ALK +. Two were RCTs [51-53], and one observational cohort study [48]. One study was excluded from the OS meta-analysis because HR survival analysis estimate was not possible since the data were presented in median-time intervals with continuous distribution [49]. Another three studies did not present any data related to the OS outcome so they were also excluded [46, 47, 50].

The analysis of the data presented by the forest plot graph for OS, expressed in HR unifying the RCT and cohort studies, demonstrated there was a beneficial trend towards the 


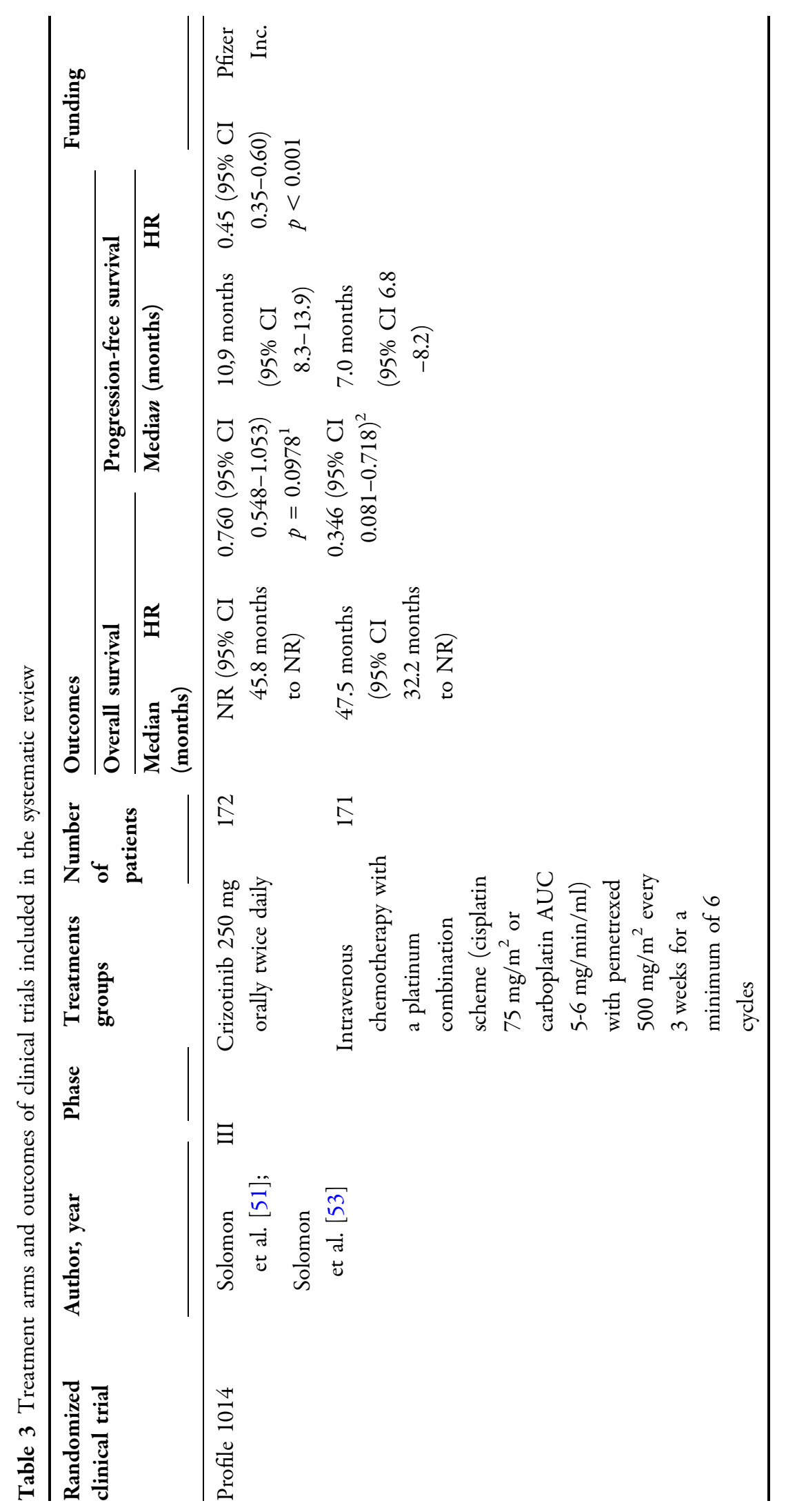




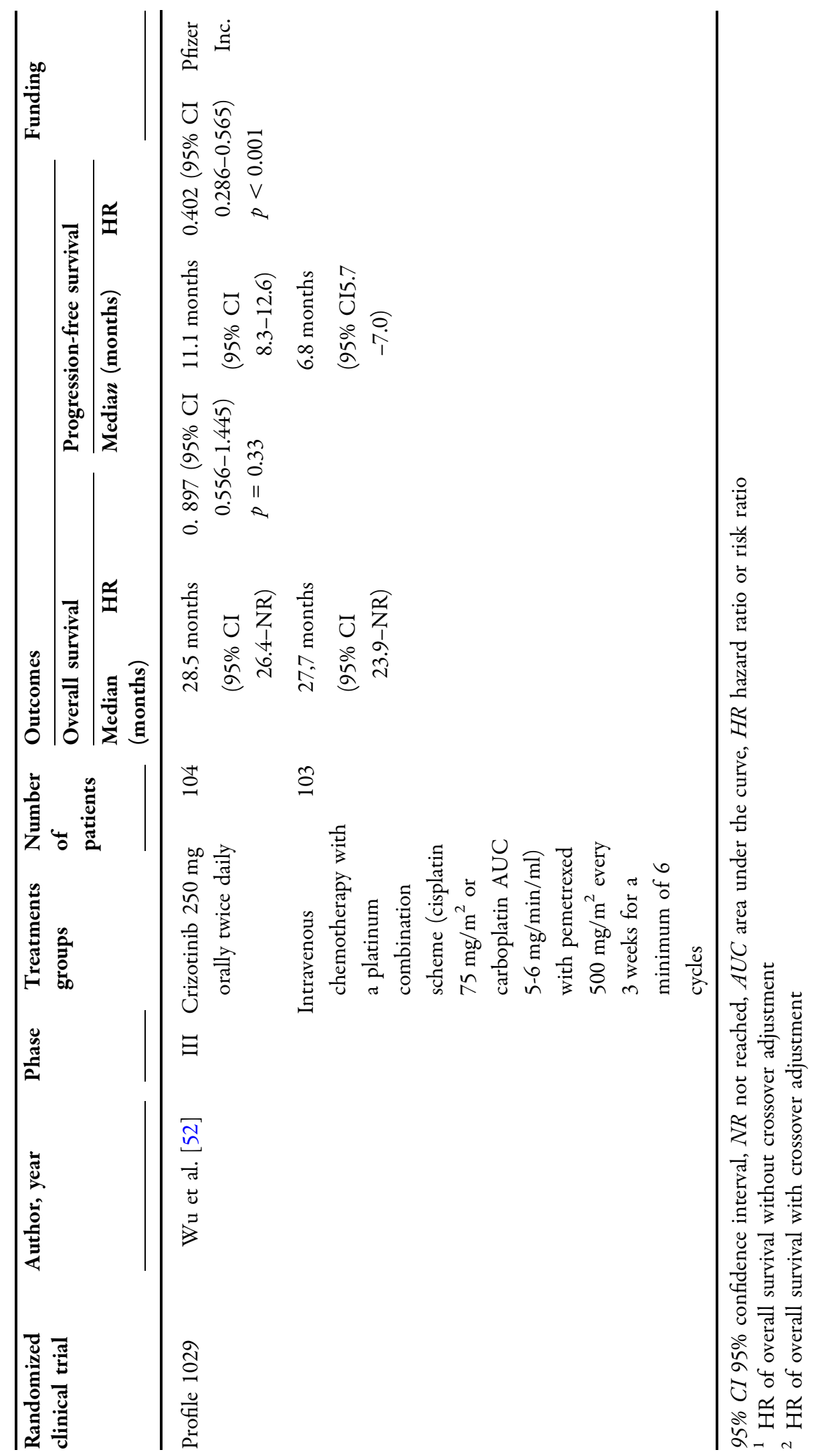




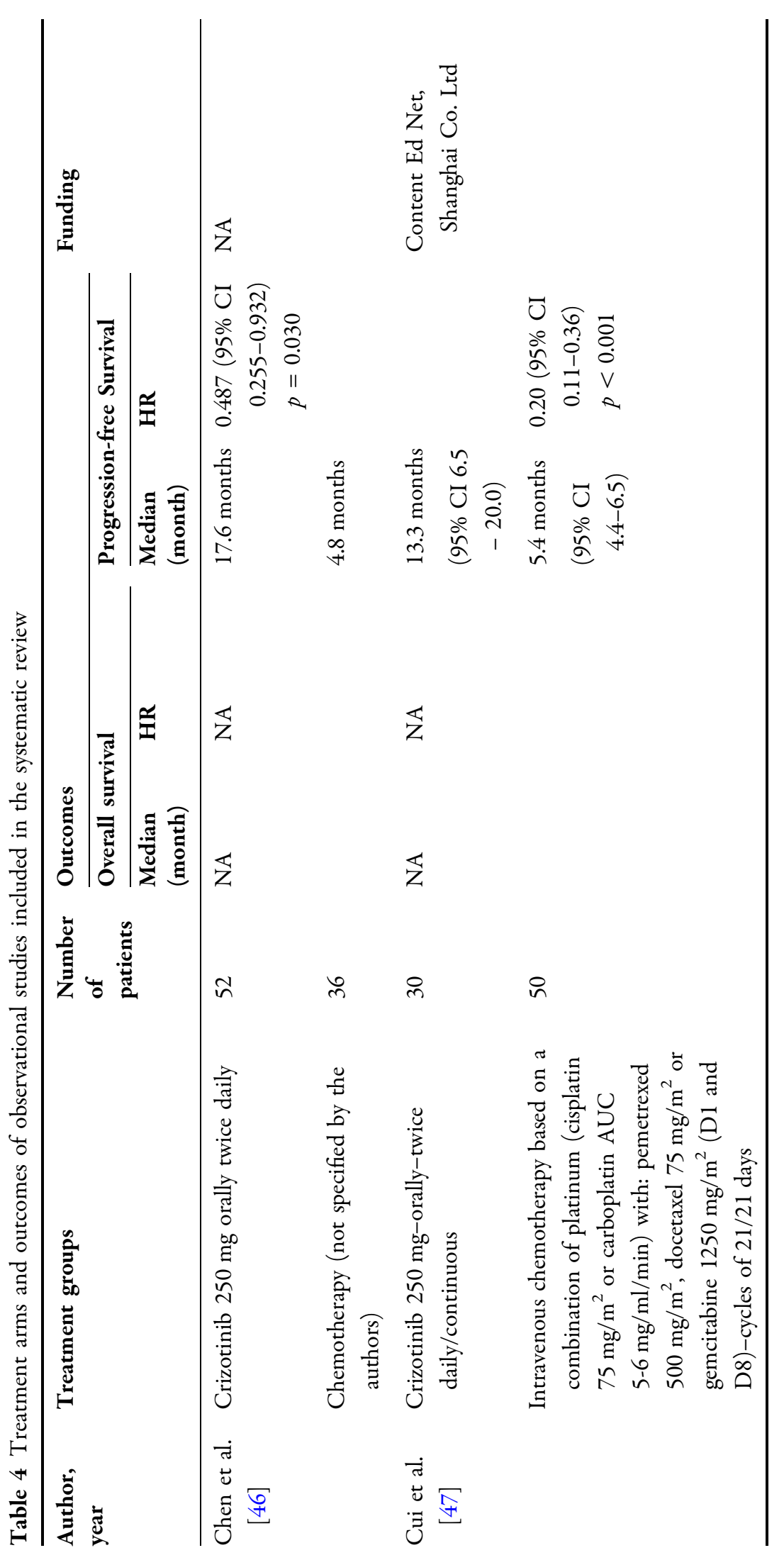




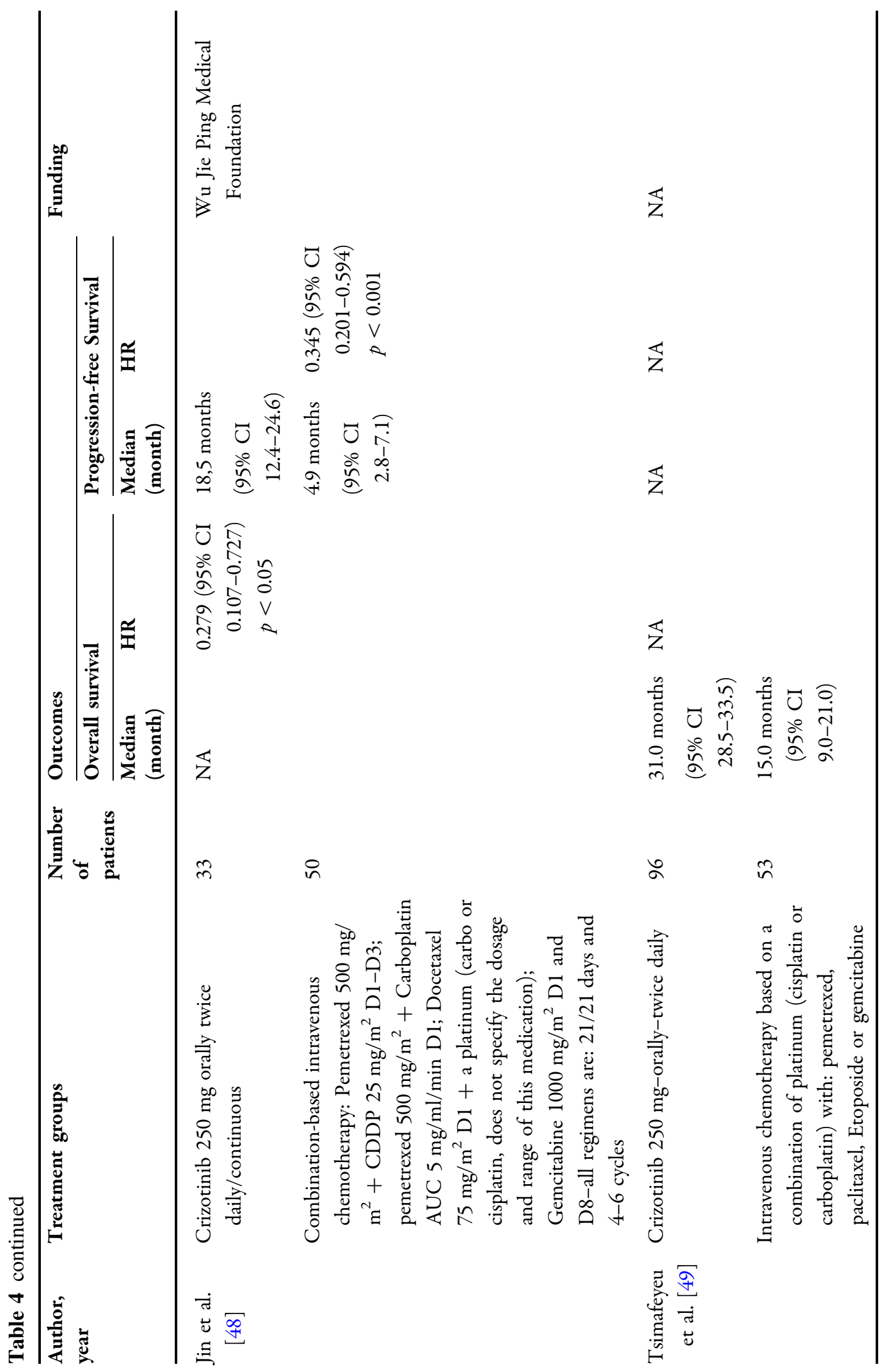




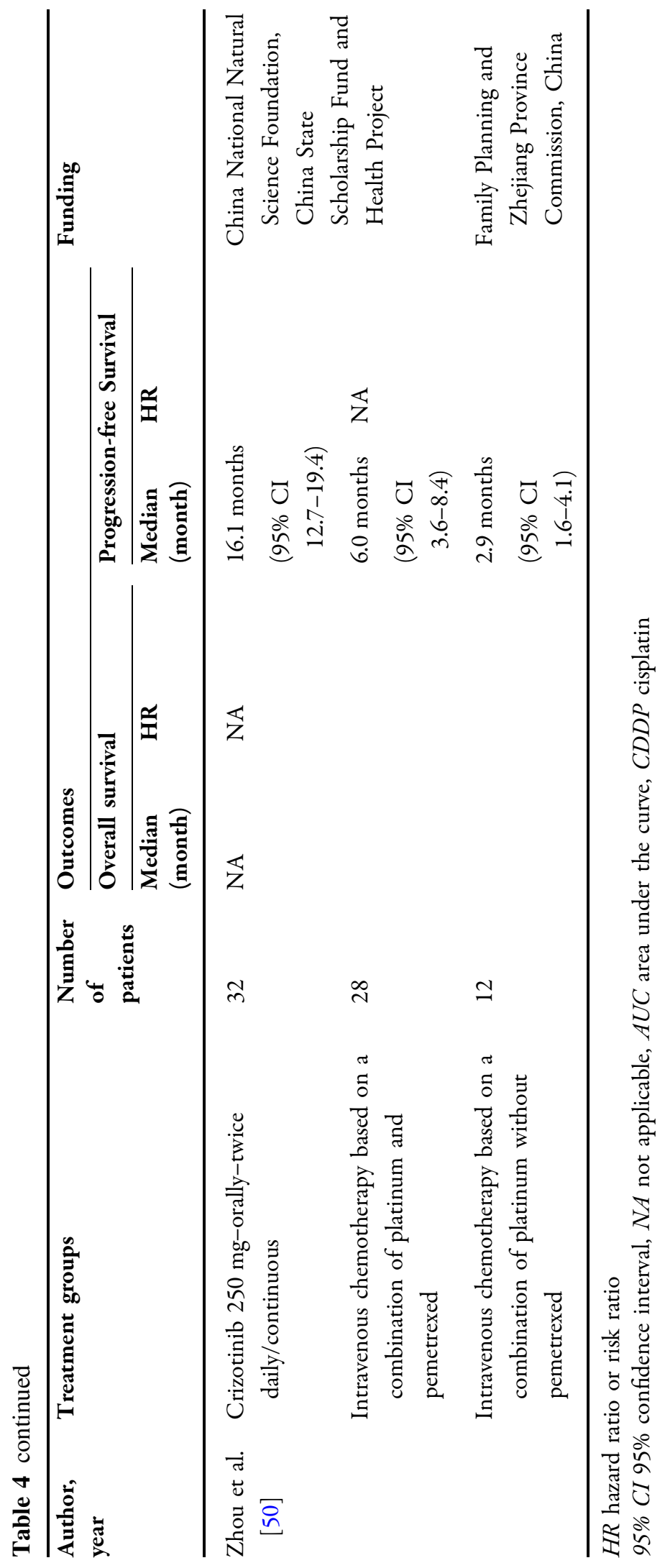




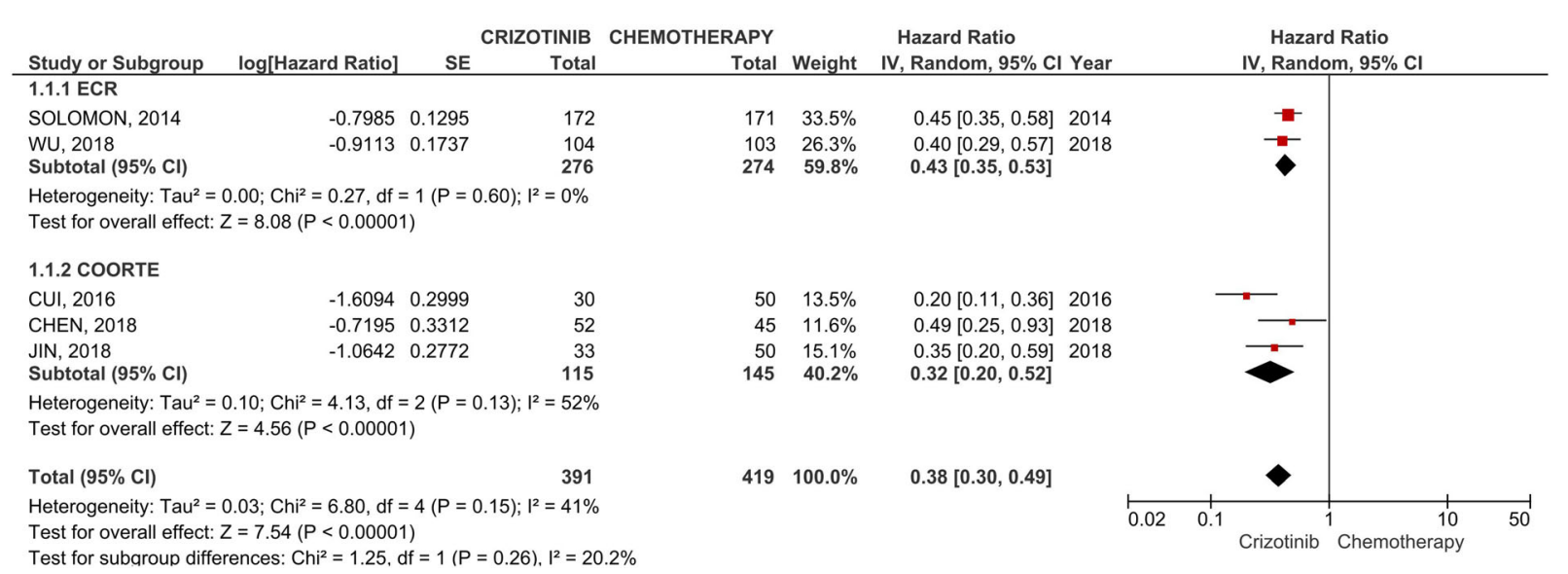

Fig. 2 Progression-free survival forest plot

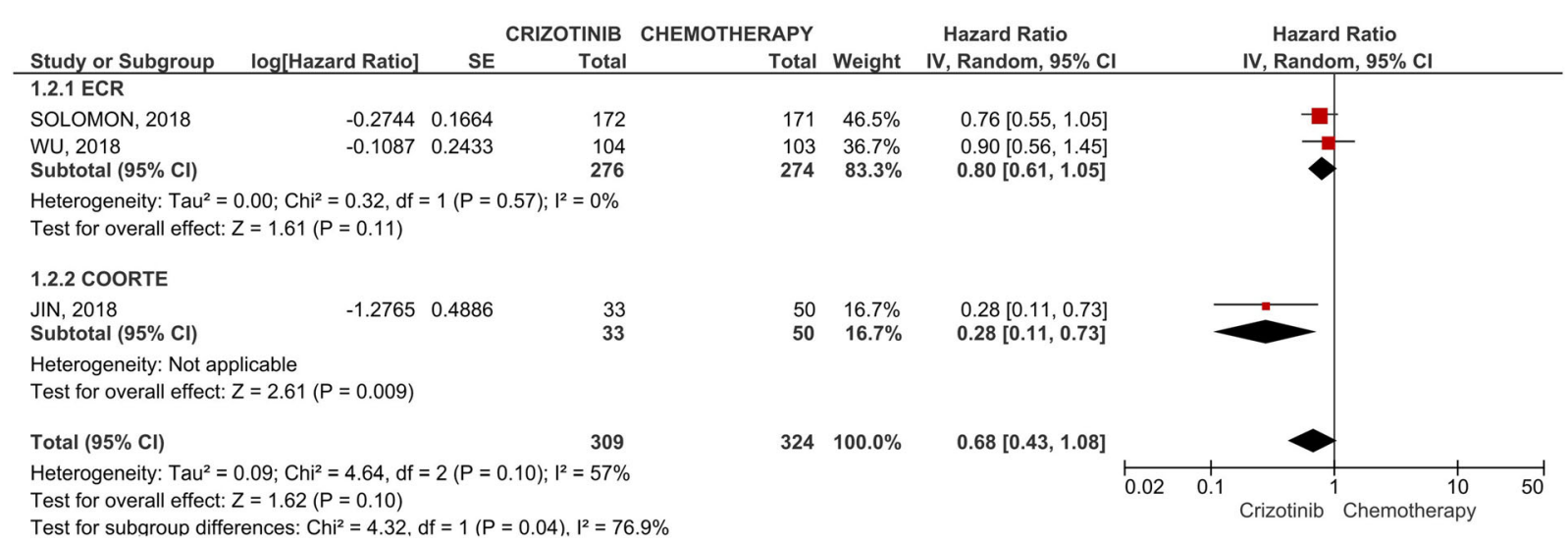

Fig. 3 Overall Survival forest plot

intervention arm (crizotinib). In contrast, the plots cross the non-effect interval, so it is not possible to affirm that the use of crizotinib will result in effective gains in overall survival- $-\mathrm{HR}$ 0.68 ; 95\% CI 0.43-1.08; $p=0.10$ (Fig. 3).

Overall, the heterogeneity from this metaanalysis is greater than desired, presenting $I^{2}=$ $57 \%$ with $p=0.10$. However, this demonstrates a higher result than previously stipulated $(p<$ $0.05)$; therefore, the extent of heterogeneity is not statistically significant. When the included studies are evaluated, it can be seen that this moderate heterogeneity is due to the methodological variation from the included studies' designs, allowing the occurrence of bias. Performing the visual inspection assessment, a greater confidence interval of the cohort study
[48] is identified with a smaller sample, thereby increasing the variance of results. When the forest plot for OS is undertaken only with ECR, the heterogeneity becomes null, but even so the diamond goes through 1 , crossing the line of no effect, demonstrating that the clinical benefit seen could be by chance rather than by the interventional drug.

\section{Methodological Quality Assessment}

The RCTs selected presented the same risk of bias profile for all domains (Fig. 4). They were characterized as uncertain for the domains of generation of the allocation sequence and allocation secrecy, and as high risk of bias for the 

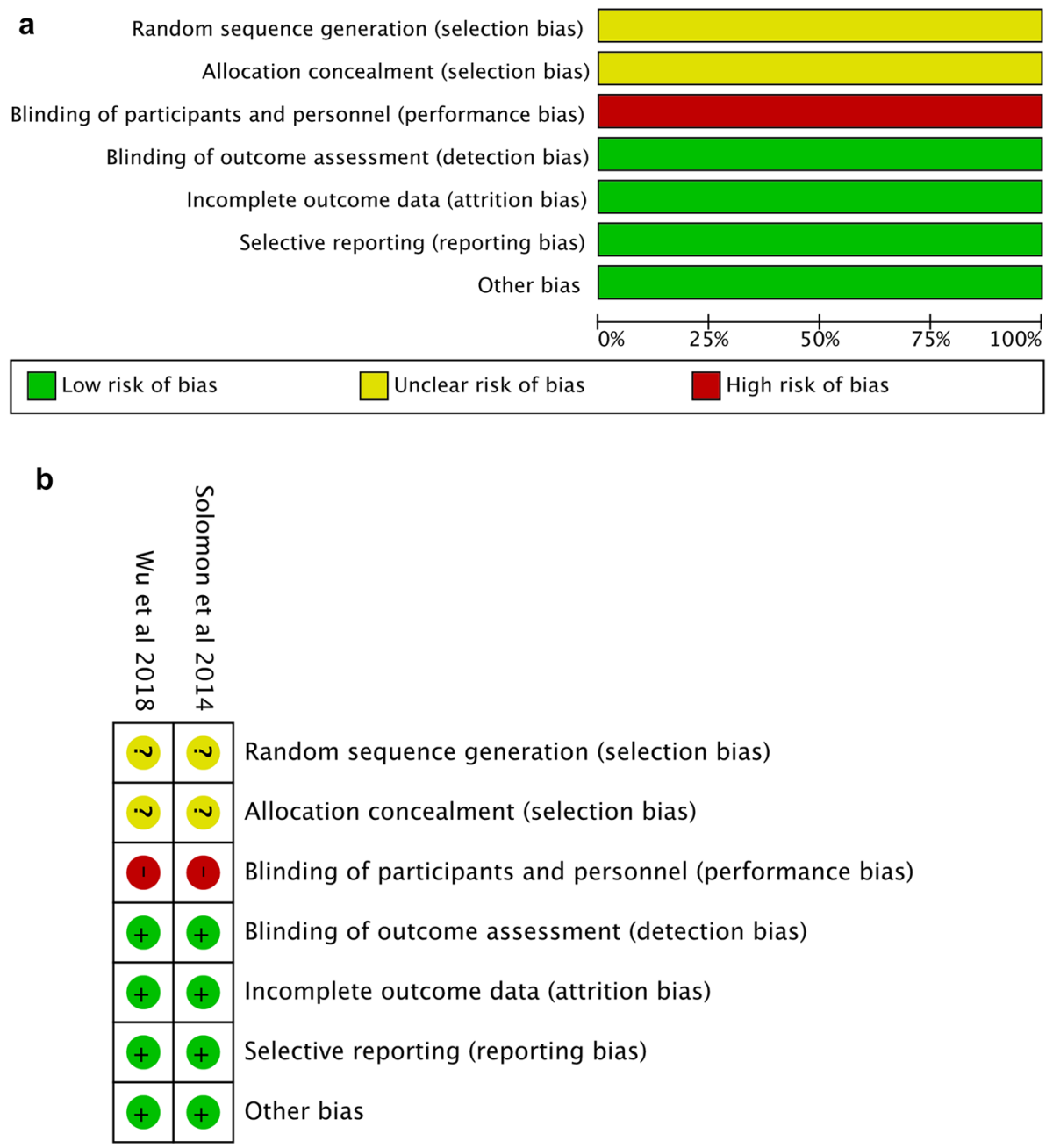

Fig. 4 Randomized controlled trials risk of bias assessment

blinding domain of the participants and staff. For the following four domains, i.e. blinding of the evaluators, incomplete outcome data, report selection of outcomes, other biases, the risk of bias was considered low [51, 52].

The five cohort studies included in the systematic review were also assessed for methodological quality using the Newcastle-Ottawa scale and all were classified as good methodological quality (Table 5).

\section{CONCLUSION}

Overall, it appeared that crizotinib improved PFS for the treatment of advanced or metastatic
NSCLC ALK+ based on HR 0.38; 95\% CI $0.30-0.49 ; p<0.001$. However, this result did not convert itself into a statistical meaningful gain in terms of an increase in OS; HR 0.68; 95\% CI $0.43-1.08 ; p=0.10$. The result crosses the non-effect interval, and has no statistical significance. Consequently, it is not possible with our study design to affirm whether there is any real benefit of using crizotinib in improving OS in this patient population. The finding results are aligned with other systematic reviews and meta-analyzes identified during the literature search [27-33].

The lack of any overall survival benefit may be due to the mechanism of patients crossing between the control and experimental 
Table 5 Cohort studies quality assessment

\begin{tabular}{lllll}
\hline Studies & Selection & Comparability & Outcome & Total \\
\hline Cui et al. [46] & ${ }^{* * *}$ & $*$ & ${ }^{* * *}$ & Good \\
Chen et al. [47] & ${ }^{* * *}$ & $*$ & $*^{* * *}$ & Good \\
Jin et al. [48] & ${ }^{* * *}$ & $*$ & $* * *$ & Good \\
Tsimafeyeu et al. [49] & ${ }^{* * *}$ & $*$ & ${ }^{* * *}$ & Good \\
Zhou et al. [50] & ${ }^{* * * *}$ & $*$ & ${ }^{* * *}$ & Good \\
\hline
\end{tabular}

treatment arms. Another possibility is that the use of subsequent therapies by the patient could also influence OS. It is not clear when analyzing the RCT data whether patients who started treatment in the control arm and migrated to therapy with experimental medication, when they were in a more advanced stage of the disease, experienced the same benefit as those who received intervention treatment from the point of randomization. In addition, crossover for OS was adjusted for patients who switched from chemotherapy to crizotinib. On the other hand, no correction was made for patients who changed from treatment with crizotinib to other subsequent therapies. All these factors can be considered plausible causes of interference in the OS results, making it impossible to perform a correct analysis of the data [51-53].

An important issue is that none of the studies showed which of the variants of ALK fusion were found in the patients, nor was a subgroup analysis of these variations performed. It is possible that this is an influencing factor in the differential response to crizotinib in non-small cell lung cancer ALK+ $[55,56]$. However, this needs further investigation before any definitive statement can be made.

Since we started our analysis, crizotinib is now available for use in private clinical practice in Brazil. However, further studies are needed to fully analyze the budgetary impact and cost-effectiveness of crizotinib within the public health system, building on the data described here, before crizotinib is funded within the public healthcare system in Brazil. Further studies may also be needed to assess possible restrictions on the use of crizotinib given some of the concerns regarding its impact on overall survival and the implications for calculating any cost/life-year saved or quality-adjusted lifeyear saved (QALY).

We are aware of a number of limitations with our systematic review and meta-analysis. Firstly, there is inevitable selection bias caused by the inclusion of only a limited number of studies in this review, preventing us from conducting any meaningful bias analyses, and only retrospective cohort studies were added. In addition, there was significant heterogeneity among the included studies, which might stem from differences in patient baseline characteristics, prior treatment regimens, and underlying genetic events. Despite these limitations, we believe our findings are robust and provide directions for future studies.

This meta-analysis demonstrated that, compared to chemotherapy, treatment with crizotinib is superior with regard to PFS in patients with non-small cell lung cancer ALK+. However, the apparent lack of an overall survival benefit probably reflects the effects of bias due to the cross-treatment allowed in randomized clinical trials and other potential limitations.

Overall, it is possible that patients with NSCLC ALK+ and good status performance do have an increase in PFS when treated with crizotinib compared to conventional chemotherapy, but without an increase in OS. Consequently, the effectiveness of crizotinib in patients with EML4-ALK-positive non-small cell lung cancer requires further confirmation of primary results, preferably with blinded designs and with subgroup stratification by genetic variations of ALK. These findings will enhance 
discussions regarding the inclusion of crizotinib within the public healthcare system of Brazil and similar LMICs.

\section{ACKNOWLEDGEMENTS}

The authors would like to thank Professor Francisco de Assis Acúrcio, Dr. Pedro Nazareth Aguiar Junior and Dr. Flávia Marini for the support, adjustments and useful advice that improved this article.

Funding. No funding or sponsorship was received for this study or publication of this article.

Authorship. All named authors meet the International Committee of Medical Journal Editors (ICMJE) criteria for authorship for this article, take responsibility for the integrity of the work as a whole, and have given their approval for this version to be published.

Author Contributions. The first draft of the manuscript was written by Barbara Delano Cruz and all authors commented on previous versions, read and approved the final manuscript. BDC contributed with the original idea, conceptualization of the study, investigation, research, data curation, formal analysis, methodology, project management, software, validation, supervision, writing, review, and editing. Material preparation, data collection and analysis were performed by BDC, Mariana Michel Barbosa, Lucas Lima Torres, Pamela Santos Azevedo, Vânia Eloisa de Araújo e Juliana Alvares-Teodoro. MMB and Brian Godman contributed with data curation, methodology, formal analysis, supervision, writing-review, and editing. LLT contributed to research, software, and validation. PSA contributed to the investigation, software, methodology, and validation. VEAS contributed to the investigation, software, methodology, and validation. JAT contributed formal analysis, methodology, project management, validation, supervision, writing, reviewing, and editing.
Disclosures. The authors (Barbara D Cruz; Mariana M Barbosa; Lucas L Torres; Pamela S Azevedo; Vânia E A Silva; Brian Godman, Juliana Alvares-Teodoro) have nothing to disclose.

Compliance with Ethics Guidelines. This article is based on previously conducted studies and does not contain any new studies with human participants or animals performed by any of the authors.

Data Availability. The datasets generated during and/or analyzed during the current study are available from the corresponding author on reasonable request.

Open Access. This article is licensed under a Creative Commons Attribution-NonCommercial 4.0 International License, which permits any non-commercial use, sharing, adaptation, distribution and reproduction in any medium or format, as long as you give appropriate credit to the original author(s) and the source, provide a link to the Creative Commons licence, and indicate if changes were made. The images or other third party material in this article are included in the article's Creative Commons licence, unless indicated otherwise in a credit line to the material. If material is not included in the article's Creative Commons licence and your intended use is not permitted by statutory regulation or exceeds the permitted use, you will need to obtain permission directly from the copyright holder. To view a copy of this licence, visit http:// creativecommons.org/licenses/by-nc/4.0/.

\section{REFERENCES}

1. Globocan. New Global Cancer Data. GLOBOCAN 2018, 2018. https://www.uicc.org/news/newglobal-cancer-data-globocan-2018.

2. IARC. World Cancer Report 2014. Editors BW Stewart and CP Wild. Available at URL: http://www. searo.who.int/publications/bookstore/documents/ 9283204298/en/.

3. Chalkidou K, Marquez P, Dhillon PK, Teerawattananon Y, Anothaisintawee T, Gadelha CA, et al. 
Evidence-informed frameworks for cost-effective cancer care and prevention in low, middle, and high-income countries. Lancet Oncol. 2014;15(3): e119-31.

4. Shah SC, Kayamba V, Peek RM, Heimburger D. Cancer control in low- and middle-income countries: is it time to consider screening? J Glob Oncol. 2019;5:1-8.

5. The L. GLOBOCAN 2018: counting the toll of cancer. Lancet. 2018;392(10152):985.

6. Dagenais GR, Leong DP, Rangarajan S, Lanas F, Lopez-Jaramillo P, Gupta R, et al. Variations in common diseases, hospital admissions, and deaths in middle-aged adults in 21 countries from five continents (PURE): a prospective cohort study. Lancet. 2020;395(10226):785-94.

7. Bray F, Ferlay J, Soerjomataram I, Siegel RL, Torre LA, Jemal A. Global cancer statistics 2018: GLOBOCAN estimates of incidence and mortality worldwide for 36 cancers in 185 countries. CA Cancer J Clin. 2018;68(6):394-424.

8. WHO. International Agency for Research on Cancer. 2020. Available at URL: https:/gco.iarc.fr/ tomorrow/home.

9. Waters R, Urquhart L. EvaluatePharma ${ }^{\circledR}$ World Preview 2019, Outlook to 2024. 2019. Available at URL: https://info.evaluate.com/rs/607-YGS-364/ images/EvaluatePharma_World_Preview_2019.pdf.

10. IMS Institute for Healthcare Informatics. Global oncology trend report. A review of 2015 and outlook to 2020. June 2016. Available at URL: https:// www.scribd.com/document/323179495/IMSHInstitute-Global-Oncology-Trend-2015-2020Report.

11. Cohen D. Cancer drugs: high price, uncertain value. BMJ (Clin Res Ed). 2017;359:j4543.

12. IQVIA. The global use of medicine in 2019 and outlook to 2023-forecasts and areas to watch. 2019. Available at URL: https://www.iqvia.com/-/media/ iqvia/pdfs/institute-reports/the-global-use-ofmedicine-in-2019-and-outlook-to-2023.pdf

13. Zheng M. Classification and pathology of lung cancer. Surg Oncol Clin N Am. 2016;25(3):447-68. https://doi.org/10.1016/j.soc.2016.02.003.

14. Duma N, Santana-Davila R, Molina JR. Non-small cell lung cancer: epidemiology, screening, diagnosis, and treatment. Mayo Clin Proc. 2019;94(8): 1623-40. https://doi.org/10.1016/j.mayocp.2019. 01.013.
15. Moldaver D, et al. Development, validation and results from the impact of treatment evolution in non-small cell lung cancer (iTEN) model. Lung Cancer. 2020;139(2019): 185-194. https://doi.org/ 10.1016/j.lungcan.2019.10.019.

16. Solomon B, Wilner KD, Shaw AT. Current status of targeted therapy for anaplastic lymphoma kinaserearranged non-small cell lung cancer. Clin Pharmacol Ther. 2014;95(1):15-23. https://doi.org/10. 1038/clpt.2013.200.

17. Elliott J, Bai Z, Hsieh S-C, Kelly SE, Chen L, Skidmore B, et al. ALK inhibitors for non-small cell lung cancer: a systematic review and network metaanalysis. PloS One. 2020;15(2):e0229179.

18. Vuong HG, Nguyen TQ, Nguyen HC, Nguyen PT, Ho ATN, Hassell L. Efficacy and safety of crizotinib in the treatment of advanced non-small-cell lung cancer with ROS1 rearrangement or MET alteration: a systematic review and meta-analysis. Target Oncol. 2020;15(5):589-98.

19. Wang M, Wang G, Ma H, Shan B. Crizotinib versus chemotherapy on ALK-positive NSCLC: a systematic review of efficacy and safety. Curr Cancer Drug Targets. 2019;19(1):41-9.

20. Kassem L, Shohdy KS, Lasheen S, Abdel-Rahman O, Ali A, Abdel-Malek RR. Safety issues with the ALK inhibitors in the treatment of NSCLC: a systematic review. Crit Rev Oncol Hematol. 2019;134:56-64.

21. Breadner D, Blanchette $P$, Shanmuganathan S, Boldt RG, Raphael J. Efficacy and safety of ALK inhibitors in ALK-rearranged non-small cell lung cancer: a systematic review and meta-analysis. Lung Cancer. 2020;144:57-63.

22. Carlson JJ, Suh K, Orfanos P, Wong W. Cost effectiveness of alectinib vs. crizotinib in first-line anaplastic lymphoma kinase-positive advanced non-small-cell lung cancer. Pharm Econ. 2018;36(4):495-504.

23. Guan H, Sheng Y, Guo W, Han S, Shi L. Cost-effectiveness of alectinib for patients with untreated ALK-positive non-small cell lung cancer in China. Adv Ther. 2019;36(5):1114-25.

24. NICE. Crizotinib for previously treated anaplastic lymphoma kinase-positive advanced non-small-cell lung cancer. Technology appraisal guidance [TA422]. 2016. Available at URL: https://www.nice. org.uk/guidance/ta422/chapter/1Recommendations.

25. SMC. Crizotinib 200mg and 250mg hard capsules (Xalkori®) SMC No 1329/18. 2018. Available at URL: https://www.scottishmedicines.org.uk/media/ 
3465/crizotinib-xalkori-final-may-2018-for-website. pdf.

26. Zampirolli Dias C, Godman B, Gargano LP, Azevedo PS, Garcia MM, Souza Cazarim M, et al. Integrative review of managed entry agreements: chances and limitations. Pharmacoeconomics. 2020;38(11): 1165-85.

27. Zhang $\mathrm{B}$, et al. Characteristics and response to crizotinib in ALK-rearranged, advanced non-adenocarcinoma, non-small cell lung cancer (NANSCLC) patients: a retrospective study and literature review. Target Oncol. 2018;13(5):631-9. https://doi.org/10.1007/s11523-018-0592-z.

28. Khan M, et al. ALK inhibitors in the treatment of ALK positive NSCLC. Front Oncol. 2019. https:// doi.org/10.3389/fonc.2018.00557.

29. Hoang T, Myung SK, Pham TT, Park B. Efficacy of crizotinib, ceritinib, and alectinib in ALK-positive non-small cell lung cancer treatment: a meta-analysis of clinical trials. Cancers (Basel). 2020;12(3). https://doi.org/10.3390/cancers12030526

30. Du X, Zheng X, Wang P, Wang Y. Efficacy and safety of crizotinib for treatment of ALK-positive NSCLC: a meta-analysis. Int J Clin Exp Med. 2018;11(11):11763-71.

31. Wang M, Wang G, Ma H, Shan B. Crizotinib versus chemotherapy on ALK-positive NSCLC: a systematic review of efficacy and safety. Curr Cancer Drug Targets. 2018;19(1):41-9. https://doi.org/10.2174/ 1568009617666170623115846.

32. Li X, et al. Efficacy and safety of crizotinib in advanced or recurrent ALK-positive non-small cell lung cancer. Chin J Lung Cancer. 2019;22(8): 488-93. https://doi.org/10.3779/j.issn.1009-3419. 2019.08.02.

33. Fan J, Fong T, Xia Z, Zhang J, Luo P. The efficacy and safety of ALK inhibitors in the treatment of ALK-positive non-small cell lung cancer: a network meta-analysis. Cancer Med. 2018;7(10):4993-5005. https://doi.org/10.1002/cam4.1768.

34. Cumpston M, Higgins JPT, Churchill R, Chandler J. Cochrane handbook for systematic reviews of interventions version 6.1.0," 2020. https://training. cochrane.org/handbook/current.

35. Moher D, Booth A, Stewart L. How to reduce unnecessary duplication: Use PROSPERO. BJOG An Int J Obstet Gynaecol. 2014;121(7):784-6. https:// doi.org/10.1111/1471-0528.12657.

36. Stewart L, Moher D, Shekelle P. Why prospective registration of systematic reviews makes sense. Syst
Rev. 2012;1(1):7-10. https://doi.org/10.1186/20464053-1-7.

37. Page MJ, Shamseer L, Tricco AC. Registration of systematic reviews in PROSPERO: 30,000 records and counting. Syst Rev. 2018;7(1):1-9. https://doi. org/10.1186/s13643-018-0699-4.

38. Liberati A, et al. The PRISMA statement for reporting systematic reviews and meta-analyses of studies that evaluate health care interventions: explanation and elaboration. PLoS Med. 2009;6(7). https:// doi.org/10.1371/journal.pmed.1000100.

39. Moher D, et al. Evaluation of ASTM standard test method E 2177, 6 retroreflectivity of pavement markings in a condition of 7 wetness. Syst Rev. 2015; $1-9$

40. Cortazar P, Zhang L, Untch M, Mehta K, Costantino JP, Wolmark N, et al. Pathological complete response and long-term clinical benefit in breast cancer: the CTNeoBC pooled analysis. Lancet. 2014;384(9938):164-72.

41. Paoletti X, Lewsley LA, Daniele G, Cook A, Yanaihara N, Tinker A, et al. Assessment of progressionfree survival as a surrogate end point of overall survival in first-line treatment of ovarian cancer: a systematic review and meta-analysis. JAMA Netw Open. 2020;3(1):e1918939.

42. Kemp R, Prasad V. Surrogate endpoints in oncology: when are they acceptable for regulatory and clinical decisions, and are they currently overused? BMC Med. 2017;15(1):134.

43. Imai $\mathrm{H}$, Mori $\mathrm{K}$, Wakuda $\mathrm{K}$, Ono A, Akamatsu $\mathrm{H}$, Shukuya T, et al. Progression-free survival, postprogression survival, and tumor response as surrogate markers for overall survival in patients with extensive small cell lung cancer. Ann Thorac Med. 2015;10(1):61-6.

44. Adunlin G, Cyrus JW, Dranitsaris G. Correlation between progression-free survival and overall survival in metastatic breast cancer patients receiving anthracyclines, taxanes, or targeted therapies: a trial-level meta-analysis. Breast Cancer Res Treat. 2015;154(3):591-608.

45. Eisenhauer EA, et al. New response evaluation criteria in solid tumours: Revised RECIST guideline (version 1.1). Eur J Cancer. 2009;45(2):228-247

46. Chen G, et al. A large, single-center, real-world study of clinicopathological characteristics and treatment in advanced ALK-positive non-small-cell lung cancer. Cancer Med. 2017;6(5):953-61. https://doi.org/10.1002/cam4.1059. 
47. Cui $\mathrm{S}$, et al. Is there a progression-free survival benefit of first-line crizotinib versus standard chemotherapy and second-line crizotinib in ALKpositive advanced lung adenocarcinoma? A retrospective study of Chinese patients. Cancer Med. 2016;5(6):1013-21. https://doi.org/10.1002/cam4. 659.

48. Jin Y, Chen Y, Yu X, Shi X. A real-world study of treatment patterns and survival outcome in advanced anaplastic lymphoma kinase-positive non-small-cell lung cancer. Oncol Lett. 2018;15(6): 8703-10. https://doi.org/10.3892/ol.2018.8444.

49. Tsimafeyeu I, et al. Overall survival of patients with ALK-positive metastatic non-small-cell lung cancer in the russian federation: nationwide cohort study. J Glob Oncol. 2019;2019(5):1-7. https://doi.org/10. 1200/JGO.19.00024.

50. Zhou J, et al. Crizotinib in patients with anaplastic lymphoma kinase-positive advanced non-small cell lung cancer versus chemotherapy as a first-line treatment. BMC Cancer. 2018;18(1):1-8. https:// doi.org/10.1186/s12885-017-3720-8.

51. Solomon BJ, et al. First-line crizotinib versus chemotherapy in ALK-positive lung cancer. N Engl J Med. 2014;371(23):2167-77.

52. Wu YL, et al. Results of PROFILE 1029, a phase III comparison of first-line crizotinib versus chemotherapy in east asian patients with ALK-positive advanced non-small cell lung cancer. J Thorac Oncol. 2018;13(10):1539-48. https://doi.org/10. 1016/j.jtho.2018.06.012.

53. Solomon BJ, et al. Final overall survival analysis from a study comparing first-line crizotinib versus chemotherapy in alk-mutation-positive non-smallcell lung cancer. J Clin Oncol. 2018;36(22):2251-8. https://doi.org/10.1200/JCO.2017.77.4794.

54. Morgan P, Woolacott N, Biswas M, Mebrahtu T, Harden M, Hodgson R. Crizotinib for untreated anaplastic lymphoma kinase-positive non-smallcell lung cancer: an evidence review group perspective of a NICE single technology appraisal. Pharmacoeconomics. 2017;35(9):909-19. https:// doi.org/10.1007/s40273-017-0497-1.

55. Cha YJ, Kim HR, Shim HS. Clinical outcomes in ALK-rearranged lung adenocarcinomas according to ALK fusion variants. J Transl Med. 2016;14(1): 1-10. https://doi.org/10.1186/s12967-016-1061-z.

56. Yoshida T, et al. Differential crizotinib response duration among ALK fusion variants in ALK-positive non-small-cell lung cancer. J Clin Oncol. 2016;34(28):3383-9. https://doi.org/10.1200/JCO. 2015.65.8732. 